\title{
Anomalies of a Nonequilibrium Spinor Polariton Condensate in a Magnetic Field
}

\author{
J. Fischer, ${ }^{1,}{ }^{*}$ S. Brodbeck, ${ }^{1}$ A. V. Chernenko, ${ }^{2}$ I. Lederer, ${ }^{1}$ A. Rahimi-Iman, ${ }^{1}$ M. Amthor, ${ }^{1}$ V. D. Kulakovskii, ${ }^{2}$ \\ L. Worschech, ${ }^{1}$ M. Kamp, ${ }^{1}$ M. Durnev,${ }^{3,4}$ C. Schneider, ${ }^{1}$ A. V. Kavokin, ${ }^{3,5}$ and S. Höfling ${ }^{1, \dagger}$ \\ ${ }^{1}$ Technische Physik, Wilhelm Conrad Röntgen Research Center for Complex Material Systems, Universität Würzburg, \\ Am Hubland, D-97074 Würzburg, Germany \\ ${ }^{2}$ Institute of Solid State Physics, Russian Academy of Sciences, Chernogolovka 142432, Russia \\ ${ }^{3}$ Spin Optics Laboratory, St-Petersburg State University, 1, Ulianovskaya, St.-Petersburg 198504, Russia \\ ${ }^{4}$ Ioffe Physical-Technical Institute of RAS, 194021 St.-Petersburg, Russia \\ ${ }^{5}$ Physics and Astronomy School, University of Southampton, Highfield, Southampton SO171BJ, United Kingdom
} (Received 15 August 2013; published 6 March 2014)

\begin{abstract}
We observe a strong variation of the Zeeman splitting of exciton polaritons in microcavities when switching between the linear regime, the polariton lasing, and photon lasing regimes. In the polariton lasing regime the sign of Zeeman splitting changes compared to the linear regime, while in the photon lasing regime the splitting vanishes. We additionally observe an increase of the diamagnetic shift in the polariton lasing regime. These effects are explained in terms of the nonequilibrium "spin Meissner effect."
\end{abstract}

The full paramagnetic screening of the Zeeman splitting in a condensate of interacting bosons, which is also referred to as the "spin Meissner effect," was predicted in 2006 [1], but no conclusive experimental evidence of this effect has been reported till now. The effect is expected to take place at thermal equilibrium and in the case of the interaction constant of bosons with parallel spins $\alpha_{1}$ exceeding the interaction constant of bosons with antiparallel spins $\alpha_{2}$ [2]. While the latter condition $\left(\alpha_{1}>\alpha_{2}\right)$ is likely to be satisfied in exciton-polariton gases in semiconductor microcavities, the former condition (thermal equilibrium) can hardly be satisfied for exciton polaritons because of their short radiative lifetime. Since 2006, several groups have reported strong nonlinear effects in magneto-optical spectra of exciton polaritons. In an exciton-polariton system, a full quenching of the Zeeman splitting has been reported by Larionov et al. [3] and Walker et al. [4]. This feature, which is characteristic for the spin Meissner effect, has, however, been accompanied by anomalies in the polarization of the polariton condensate, leading to an alternative interpretation of the observed quenching. Recently, Gorbunov et al. have reported the reduction of Zeeman splitting with the increase of pumping in a system of spatially indirect excitons, whose radiative lifetime exceeds by several orders of magnitude the lifetime of exciton polaritons [5]. Polariton condensates are far more out of equilibrium than indirect excitons, which makes an accurate theoretical description of the according Zeeman splitting highly nontrivial. In the experiment by Walker et al. [4] the observed effects were explained in terms of an interplay of selfinduced Larmor precession, optical anisotropy, and phase synchronization rather than the spin Meissner effect. Korenev [6] has proposed a kinetic model to describe the experiments by Larionov et al. [3] based on a nonstandard assumption of attraction of polaritons with parallel spins. Meanwhile, the full quenching of the Zeeman splitting has also been observed in electrically pumped polariton lasers below a critical magnetic field [7].

In this work we present a systematic study of the Zeeman splitting, circular polarization degree, and diamagnetic shift of the cavity polariton mode in the low excitation regime, polariton lasing regime, and photon lasing regime above the Mott density in the quantum wells (QWs). We show that the observed features can be qualitatively explained within the same model based on the assumption of thermal equilibrium within each of the polariton spin components, but no equilibrium between two spin components. We argue that this regime is likely to be observed in spinor bosonic systems where the energy relaxation rate strongly exceeds the spin relaxation rate.

The studied microcavity is a high- $Q(\sim 10000)$ FabryPérot microresonator grown by molecular beam epitaxy (Supplemental Material [8]). All experiments were carried out under nonresonant pumping with a top-hat shaped laser spot of $40 \mu \mathrm{m}$ diameter and at a temperature of about $T \approx 5 \mathrm{~K}[8]$.

The first part of our study addresses the power dependent emission characteristics of our device. By varying the excitation power, we can identify the three regimes of linear polaritons [Fig. 1(a)], polariton condensate [Fig. 1(b)], and weak coupling lasing [Fig. 1(c)]. The third regime (photon lasing) can be reached only under pulsed excitation (pulse width $\sim 50 \mathrm{ps}$ ), due to limited cw-excitation powers (see [8] for power series under pulsed excitation). In Figs. 1(d)-(f) we plot the input-output characteristics, the power dependent energy and linewidth of the $k_{\|}=0$ ground state, which are in good agreement with literature $[9,10]$, indicating the 

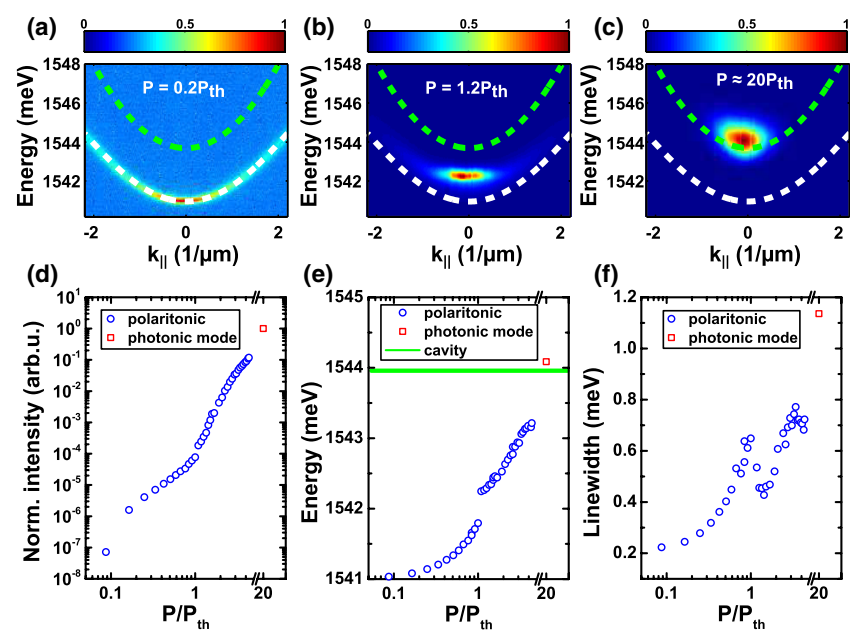

FIG. 1 (color online). Power series for detuning $\delta=$ $-6.5 \mathrm{meV}$. (a)-(c) Fourier-space-resolved (momentum-space $[8,11])$ spectra of the polariton emission at power $P=0.2 P_{\text {th }}$, $P=1.2 P_{\text {th }}$ and $P \approx 20 P_{\text {th }}$. The white dashed line marks the theoretic polariton dispersion for this detuning, the green dashed line indicates the photonic dispersion. The diagram (d) presents the input-output curve, where the power is normalized to the threshold power $P_{\text {th }}=5 \mathrm{~mW}$. The blue circles are the intensity values for continuous wave excitation and the red square corresponds to photon lasing under pulsed excitation. (e) and (f) show the energy shift and the linewidth versus excitation power.

crossover between the three different regimes of the microcavity (see Ref. [8] for more detailed discussion).

Next, we investigate the interaction of the lower polaritons (LPs) in the linear regime with an external magnetic field. The latter is applied along the growth direction of the sample, which is the so-called Faraday geometry. Figure 2(a) depicts the Zeeman splitting $\Delta E_{Z}$ as a function of the applied magnetic field from $B=0 \mathrm{~T}$ to $B=5 \mathrm{~T}$ for three different detunings $\delta=-8.9 \mathrm{meV}$, $\delta=-4.7 \mathrm{meV}$ and $\delta=+2.7 \mathrm{meV}$. The detunings are determined at zero magnetic field; however, they change for higher magnetic fields due to the excitons' diamagnetic shift [8]. In the case of Fig. 2(a) the detuning $\delta=$ $+2.7 \mathrm{meV}$ at $B=0 \mathrm{~T}$ decreases to $\delta=+1.6 \mathrm{meV}$ at $B=5 \mathrm{~T}$ resulting in the nonlinear behavior of the Zeeman splitting in Fig. 2(a). This nonlinearity is especially pronounced for magnetic fields $B>2.5 \mathrm{~T}$ via a flattening of the mode splitting, and for measurements in the zero and blue detuning regime due to a larger relative change in the Hopfield coefficient. Furthermore, it is well known that the exciton $g$ factor itself has a dependency on the magnetic field [12], which is in particular pronounced for wide QWs [13]. Therefore, for the sake of consistency, we compare the extracted Zeeman splittings of the LP recorded at a magnetic field of $B=5 \mathrm{~T}$ as a function of the cavityexciton detuning in Fig. 2(b). According to previous investigations the Zeeman splitting in the linear regime can be approximated by the following expression [14]:
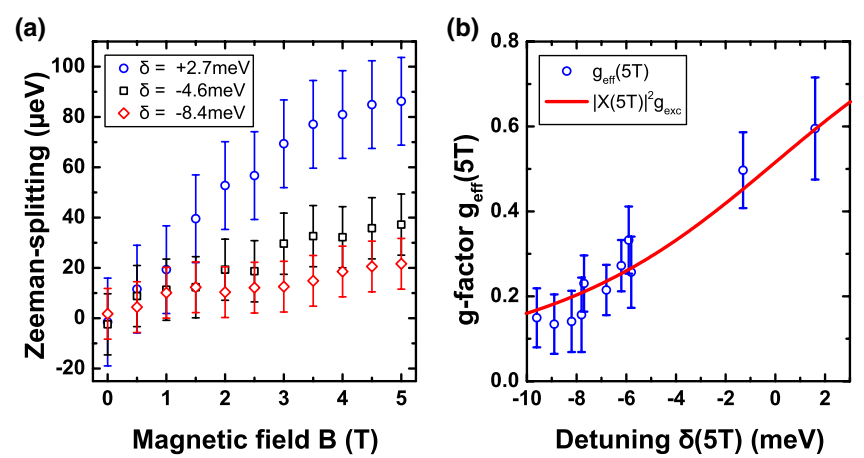

FIG. 2 (color online). (a) Zeeman splittings for three different representative detunings $\delta=+2.7 \mathrm{meV}, \delta=-4.6 \mathrm{meV}$, and $\delta=-8.4 \mathrm{meV}$ magnetic field. With increasing excitonic part $|X|^{2}$ (from negative to positive detuning) the Zeeman splitting increases from minimal value $\Delta E_{Z}=18 \mu \mathrm{eV}$ at $\delta=-9.6 \mathrm{meV}$ $(B=5 \mathrm{~T})$ to $\Delta E_{Z}=86 \mu \mathrm{eV}$ at $\delta=+1.6 \mathrm{meV} \quad(B=5 \mathrm{~T})$. (b) Extracted $g$ factor of the LP in the linear regime at $B=$ $5 \mathrm{~T} g_{\text {eff }}(B=5 T)$ plotted versus detuning. The red line shows the expected theoretical behavior.

$$
\begin{aligned}
\Delta E_{Z}\left(\left|X(\delta, B)_{k_{\|}}\right|^{2}, B\right) & =\left|X(\delta, B)_{k_{\|}}\right|^{2} g_{X} \mu_{B} B \\
& =g_{\mathrm{eff}} \mu_{B} B,
\end{aligned}
$$

$\mu_{B}$ is the Bohr magneton, $B$ the applied magnetic field along the growth axis, and $\left|X(\delta, B)_{k_{\|}}\right|^{2}$ describes the matter part of the polariton (Hopfield coefficient). The solid red line in Fig. 2(b) shows the fitting results, which can accurately reproduce the measured data for an exciton $g$ factor $g_{X}(5 T)$ of approximately 1 .

We now assess the fundamental differences in the response of our system on the magnetic field when different operating regimes of the MC are considered: Figs. 3(a)-(c) depict the dispersions at $B=0 \mathrm{~T}$ (left) and $B=5 \mathrm{~T}$ (right) for three representative excitation powers to illustrate the diamagnetic shift of the polariton emission. In Fig. 3(a) one can note a shift of the emission energy throughout the entire dispersion. The white line indicates hereby the LP dispersion, the green dashed line the photon-mode, and the red dashed one the excitonic energy. Because of the magnetic field influence on the oscillator strength of the QWs, the Rabi splitting increases from $E_{\mathrm{RS}}=10.1 \mathrm{meV}$ to about $E_{\mathrm{RS}}=10.5 \mathrm{meV}$ [15] and the exciton energy shifts about $\Delta E_{\mathrm{dia}, X}=+1.2 \mathrm{meV}$, while the photonic mode is not affected. This results in a change of the detuning from $\delta=-6.5 \mathrm{meV}$ to $\delta=-7.7 \mathrm{meV}$ (see [8] for more details). In Figs. 3(d)-(f) waterfall diagrams of the right-handed circular polarized $\left(\sigma^{+}\right)$and left-handed circular polarized $\left(\sigma^{-}\right)$component are plotted for each power from $B=0 \mathrm{~T}$ to $B=5 \mathrm{~T}$ to comparably show the magnetic field influence on the mode splitting. The line spectra are taken from the far field images by integrating around the ground state in an interval of $k_{\|}= \pm 0.25(1 / \mu \mathrm{m})$. Figure 3(d) shows the dependence of the uncondensed LP on the 

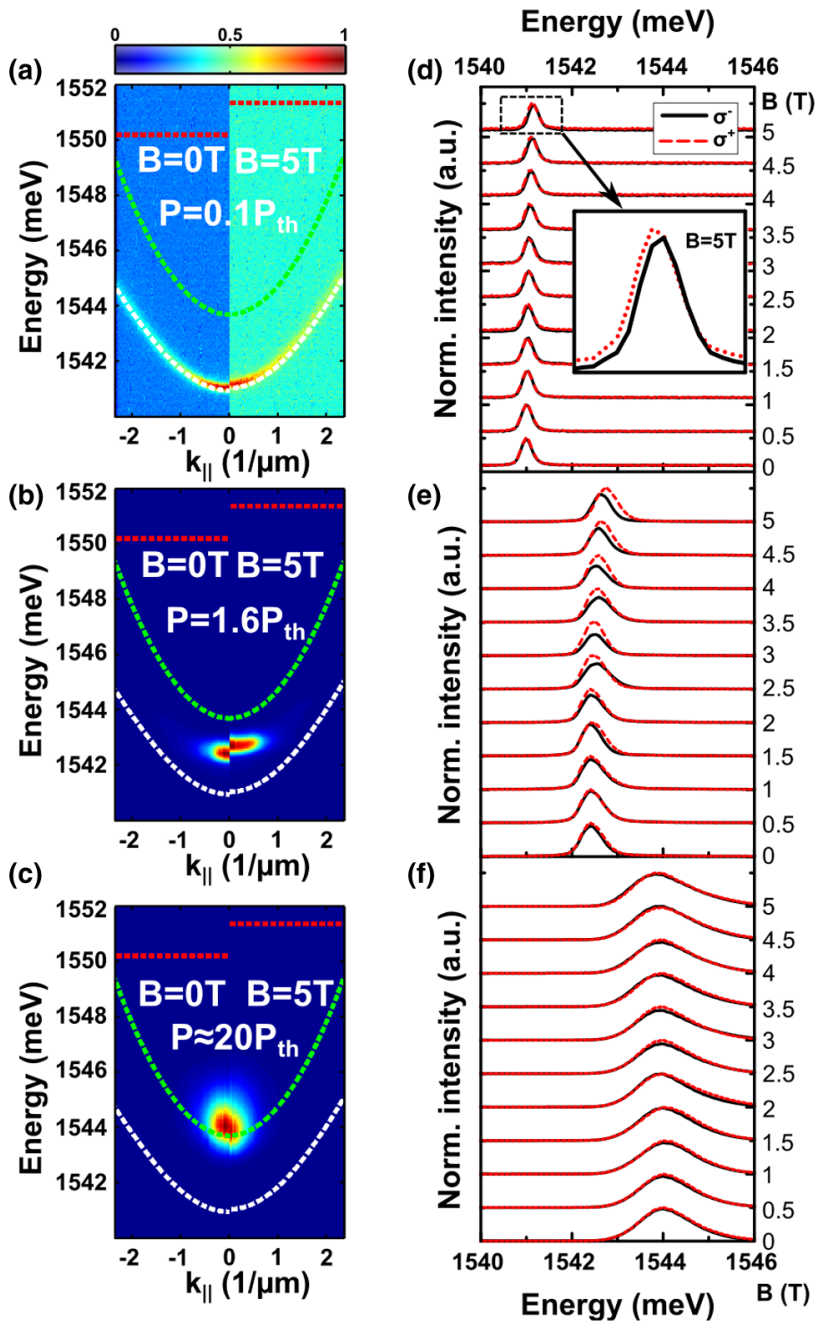

FIG. 3 (color online). (a)-(c) Fourier-space spectra for $B=0 \mathrm{~T}$ and $B=5 \mathrm{~T}$ (left and right side) for the three different operation regimes of the microcavity at the excitation powers $P=0.1 P_{\mathrm{th}}$, $P=1.6 P_{\text {th }}$ and $P \approx 20 P_{\text {th }}$, showing the mode shift in magnetic fields (diamagnetic shift). (d)-(f) show polarization resolved spectra to depict the energy of the ground state (black line $\sigma^{-}$ and red dashed line $\sigma^{+}$) for the same excitation powers as in (a)-(c). For the lower polariton and the polariton condensate (d),(e) a Zeeman splitting is observable. In the photonic lasing regime (f) the mode splitting is absent. Also no differences in the intensity are visible.

magnetic field. The inset illustrates a small Zeeman splitting $\Delta E_{Z}=22 \mu \mathrm{eV}$ at $B=5 \mathrm{~T}$, consistent with our analysis in Fig. 2(b). Above the polariton laser threshold in Fig. 3(b) and Fig. 3(e), the Zeeman splitting preserves a small value to a critical magnetic field $B_{C}$ and increases linearly with $B$ above 3 T. We furthermore observe a significantly larger diamagnetic shift of the polariton emission in this pumping regime. In stark contrast, any diamagnetic shift or Zeeman splitting is entirely absent in the cavity mediated photonic lasing regime, as shown in Fig. 3(c) and Fig. 3(f). Despite previous observations of weak responses of vertical cavity surface emitting lasers on axial magnetic fields [16] in terms of their polarization and mode energy, this strongly suggests that the polaritonic origin of the emission is completely lost. We note, that this strong and fundamental difference of the response of the photon and polariton laser on the magnetic field is a supreme tool to distinguish between the two phases [7], even if other properties such as dispersion, coherence, linewidth trace, occupation, and energy indeed can share many similarities $[17,18]$.

The values extracted from the spectra in Figs. 3(d) and 3(e) are plotted in Figs. 4(a) and 4(b) as functions of the applied magnetic field. The small, rather linear increase of the Zeeman splitting in the linear regime follows the trend discussed above in Fig. 2(b), resulting in an effective $g$ factor $g_{\text {eff }}$, as small as +0.16 due to the large photonic content. In the polariton lasing regime, the Zeeman splitting cannot be experimentally resolved up to a magnetic field of $B_{C} \sim 3 \mathrm{~T}$, as shown in Fig. 4(a). Remarkably, the Zeeman-splitting changes its sign above this critical field and its magnitude is significantly increased with respect to the uncondensed polariton Zeeman splitting.

Taking a fully thermalized system into account as proposed in Ref. [1], the measured sign reversal of the $g$ factor is not predicted (see Supplemental Material, Fig. 3 [8]). We reproduce this experimental data within a model, which extends the theory by Rubo et al. [1] by implying the thermal equilibrium within each spin component of the exciton-polariton gas, but no thermal equilibrium between spin-up and spin-down polaritons. This corresponds to the regime of fast energy relaxation due to polariton-phonon interactions, but slow spin relaxation. In our case the data are recorded at a red detuning of about $\delta=-6.5 \mathrm{meV}$. For such a detuning, the spin relaxation time of exciton polaritons is in the region of 300 ps [19], which is much longer than the energy relaxation time (in the order of a few tens of ps) [20].
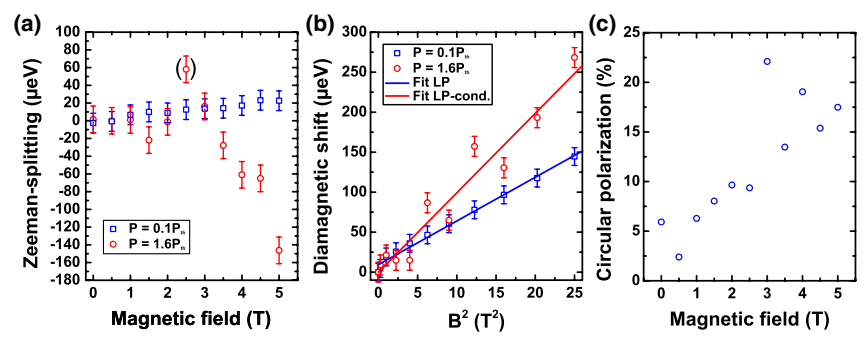

FIG. 4 (color online). Measured Zeeman splitting and diamagnetic shift for $P=0.1 P_{\text {th }}$ (blue squares) and $P=1.6 P_{\text {th }}$ (red dots). In (a) the Zeeman splitting for the lower polariton shows the previous discussed linear behavior, while the condensate mode splitting is characterized by a sign reversal of the Zeeman splitting. (b) The diamagnetic shift for both regimes is depicted as a function of the applied magnetic field. In (c) we plot the degree of linear polarization extracted from the spectra of Fig. 3(e). 
Consider a localized condensate of interacting exciton polaritons subjected to the magnetic field $B$ at zero temperature. Its free energy reads (for more details of the model, see [8]):

$$
\begin{aligned}
F= & -\mu_{+} n_{+}-\mu_{-} n_{-}+\frac{\alpha_{1}+\alpha_{2}}{4} n^{2}+\left(\alpha_{1}-\alpha_{2}\right) S_{z}^{2} \\
& -\mu_{B} g_{\mathrm{eff}} B S_{z} .
\end{aligned}
$$

Here $n=n_{+}+n_{-}$is the number of polaritons, $n_{+}$and $n_{-}$ are the numbers of $\sigma^{+}$-and $\sigma^{-}$-polarized polaritons and $S_{z}=\left(n_{-}-n_{+}\right) / 2$. The interaction constants for polaritons with parallel and antiparallel spins are denoted as $\alpha_{1}$ and $\alpha_{2}$, while the chemical potentials for $\sigma^{+}$-and $\sigma^{-}$-polarized polaritons are $\mu_{+}$and $\mu_{-}$, which may not be identical (see below). In the conventional case of microcavity polaritons, the interaction constants satisfy the following condition:

$$
\alpha_{1}-\alpha_{2}>0
$$

Assuming out of equilibrium conditions between the two spin subsystems, the minimum of the free energy is achieved at

$$
S_{z}=\frac{n}{2} \rho_{c}=\frac{\mu_{B} g_{\mathrm{eff}} B-\Delta}{2\left(\alpha_{1}-\alpha_{2}\right)},
$$

where $\Delta=\mu_{+}-\mu_{-}$is the Zeeman splitting. From Eq. (4) one can express:

$$
\Delta=\mu_{B} g_{\mathrm{eff}} B-\left(\alpha_{1}-\alpha_{2}\right) n \rho_{c} .
$$

One can see that the sign of $\Delta$ may be negative if $\left(\alpha_{1}-\alpha_{2}\right) n \rho_{c}>\mu_{B} g_{\text {eff }} B$. Figure $5(\mathrm{a})$ shows the Zeeman splitting at low $(n \rightarrow 0)$ and high power calculated according to the expression Eq. (5). The assumed dependence of the circular polarization degree as a function of magnetic field at the high power is shown in Fig. 5(c). It nicely compares with the experimental data on the circular
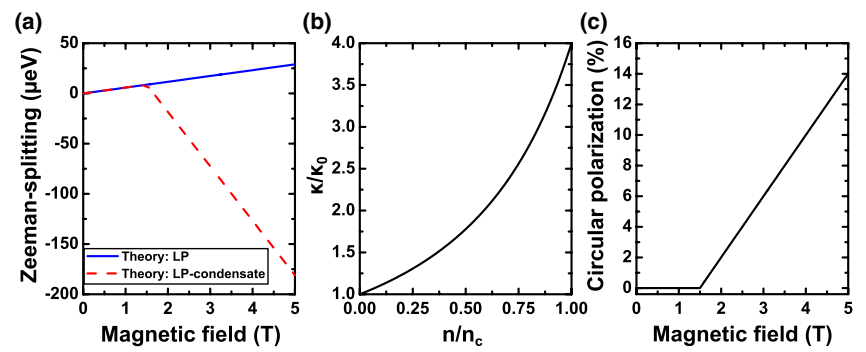

FIG. 5 (color online). (a) The theoretical fit of the Zeeman splitting using the dependence of the circular polarization of emission on the magnetic field shown in (c). (b) Relative change of the diamagnetic coefficient as a function of the exciton-density in the quantum well. $n_{c}$ corresponds to the expected Mott density and $\kappa_{0}$ is the diamagnetic shift for low excitation powers. polarization degree of the photoluminescence extracted from Fig. 3(e). It follows directly from Eq. (5), if the polarization degree is zero, linear and nonlinear theories predict the same Zeeman splitting [see Fig. 5(a)]. In a more phenomenological picture, the quasiequilibrium counterpart of the spin Meissner effect can be understood as interplay between magnetic orientation of polariton spins and a density dependent blueshift of each level. In the original theory, via increasing $B$, the polaritons are redistributed between two Zeeman levels in order to keep constant the chemical potential of the condensate. This is accompanied by a full quenching of the Zeeman splitting until a critical field $B_{C}$ is reached $[1,8]$. In our quasiequilibrium model, the relative occupation of the two Zeeman levels is not governed by thermal equilibrium, it is dependent on the polarization degree of the condensate: an independent parameter which can be assessed experimentally [Fig. 4(c)]. As a result, we still observe a competition between Zeeman splitting and interaction blueshifts, which consistently explains the experimental features in Fig. 4(a). Note that the present model would not explain the observations of Larionov et al. [3] who detected zero Zeeman splitting and strong negative circular polarization at the same pumping intensity. This indicates that the condition of quasiequilibrium is probably not fulfilled in the experiment of Larionov. Now we compare the effective diamagnetic coefficients $\kappa_{\text {eff }}$ of both regimes in Fig. 4(b) by fitting the data with $\Delta E_{\mathrm{dia}}(B)=\kappa_{\mathrm{eff}} B^{2}$. Both, the linear lower polariton and the condensate are subject to a diamagnetic shift, which is characterized by the coefficient of $\kappa_{\text {eff,LP }}=6\left(\mu \mathrm{eV} / \mathrm{T}^{2}\right)$ for the LP and $\kappa_{\text {eff,con }}=$ $10\left(\mu \mathrm{eV} / \mathrm{T}^{2}\right)$ for the condensate. The increase of the diamagnetic coefficient in the condensate regime can be understood by taking into account the effects of phasespace filling in our nonresonantly driven system. Upon increasing the pump power, exciton screening results in an increase of the exciton Bohr radius $a_{B}=\left(4 \pi \epsilon \epsilon_{0} \hbar^{2} / 2 \mu e^{2}\right)$, and conclusively in an increased diamagnetic coefficient $\kappa$ [21] (Supplemental Material [8]).

In our case the diamagnetic shift increases by a factor of 1.67. Assuming this density dependence of the latter, we can extract an exciton density of $n=0.45 n_{C}$ [Fig. 5(b)]. This matches the polariton density, which we used when describing the Zeeman splitting and circular polarization of polariton emission at this pumping power. Clearly, the three experimental features of polariton gases in the presence of the external magnetic field (Zeeman splitting, circularity and diamagnetic shift) can be explained within a single model. Using the value $a_{B}=10 \mathrm{~nm}$ we obtain $n_{c}=$ $6.4 \times 10^{11} \mathrm{~cm}^{-2}$ and $n=2.9 \times 10^{11} \mathrm{~cm}^{-2}$. It yields $\alpha_{1}-\alpha_{2}=5.2 \times 10^{-12} \mathrm{meV} \mathrm{cm}^{2}$ in reasonable agreement with other experiments [22,23].

In conclusion, each of three operating regimes of our microcavity device is characterized by a peculiar response to the magnetic field: A conventional Zeeman splitting 
proportional to the excitonic content for polaritons in the linear regime, a quenching of the Zeeman splitting at low magnetic field in the polariton condensate regime with a unique sign reversal of the $g$ factor, and no notable magnetic field response in the weak coupling regime. These observations have been interpreted within the quasiequilibrium model, which assumes the thermal equilibrium within each Zeeman component of the polariton cloud, but no thermal equilibrium between two Zeeman components. The nonequilibrium spin Meissner effect taking place in this regime manifests itself in the inversion of the sign of the Zeeman splitting of a polariton condensate. Our experimental technique can be considered as a unique and powerful tool to unambiguously discriminate a polariton laser from a cavity mediated laser in the weak coupling regime, which has been a topic attracting lots of scientific interest and disputes.

The authors would like to thank the State of Bavaria for financial support. M. D. and A. V. K. acknowledge support from the Russian Ministry of Science and Education (Contract No. 11.G34.31.0067). A. V. C. acknowledges support from the Russian Foundation for Basic Research.

*julian.fischer@physik.uni-wuerzburg.de

${ }^{\dagger}$ Present address: SUPA, School of Physics and Astronomy, University of St Andrews, St Andrews KY16 9SS, United Kingdom.

sh222@st-andrews.ac.uk

[1] Y. G. Rubo, A. V. Kavokin, and I. A. Shelykh, Phys. Lett. A 358, 227 (2006).

[2] M. Vladimirova, S. Cronenberger, D. Scalbert, K. V. Kavokin, A. Miard, A. Lemaître, J. Bloch, D. Solnyshkov, G. Malpuech, and A. V. Kavokin, Phys. Rev. B 82, 075301 (2010).

[3] A. V. Larionov, V. D. Kulakovskii, S. Höfling, C. Schneider, L. Worschech, and A. Forchel, Phys. Rev. Lett. 105, 256401 (2010).

[4] P. Walker, T. C. H. Liew, D. Sarkar, M. Durska, A. P. D. Love, M. S. Skolnick, J. S. Roberts, I. A. Shelykh, A. V. Kavokin, and D. N. Krizhanovskii, Phys. Rev. Lett. 106, 257401 (2011)

[5] A. V. Gorbunov, and V. B. Timofeev, Solid State Commun. 157, 6 (2013).

[6] V. L. Korenev, Phys. Rev. B 86, 035310 (2012).

[7] C. Schneider, A. Rahimi-Iman, N. Y. Kim, J. Fischer, I. G. Savenko, M. Amthor, M. Lermer, A. Wolf, L. Worschech,
V. D. Kulakovskii, I. A. Shelykh, M. Kamp, S. Reitzenstein, A. Forchel, Y. Yamamoto, and S. Höfling, Nature (London) 497, 348 (2013).

[8] See Supplemental Material at http://link.aps.org/ supplemental/10.1103/PhysRevLett.112.093902 for details of the sample design, excitation scheme, pulsed power series and a derivation of the theory applied in the main manuscript.

[9] J. Kasprzak, M. Richard, S. Kundermann, A. Baas, P. Jeambrun, J. M. J. Keeling, F. M. Marchetti, M. H. Szymańska, R. André, J. L. Staehli, V. Savona, P. B. Littlewood, B. Deveaud, and L. S. Dang, Nature (London) 443, 409 (2006).

[10] R. Balili, V. Hartwell, D. Snoke, L. Pfeiffer, and K. West, Science 316, 1007 (2007).

[11] C. W. Lai, N. Y. Kim, S. Utsunomiya, G. Roumpos, H. Deng, M. D. Fraser, T. Byrnes, P. Recher, N. Kumada, T. Fujisawa, and Y. Yamamoto, Nature (London) 450, 529 (2007).

[12] S. N. Walck, and T. L. Reinecke, Phys. Rev. B 57, 9088 (1998).

[13] M. J. Snelling, E. Blackwood, C. J. McDonagh, R. T. Harley, and C. T. B. Foxon, Phys. Rev. B 45, 3922 (1992).

[14] A. Rahimi-Iman, C. Schneider, J. Fischer, S. Holzinger, M. Amthor, S. Höfling, S. Reitzenstein, L. Worschech, M. Kamp, A. Forchel, Phys. Rev. B 84, 165325 (2011).

[15] T. A. Fisher, A. M. Afshar, M. S. Skolnick, D. M. Whittaker, and J. S. Roberts, Phys. Rev. B 53, R10469 (1996).

[16] A. K. Jansen van Doorn, M. P. van Exter, M. Travagnin, and J. P. Woerdman, Opt. Commun. 133, 252 (1997).

[17] H. Ohadi, E. Kammann, T. C. H. Liew, K. G. Lagoudakis, A. V. Kavokin, and P. G. Lagoudakis, Phys. Rev. Lett. 109, 016404 (2012).

[18] D. Bajoni, P. Senellart, A. Lemaître, and J. Bloch, Phys. Rev. B 76, 201305 (2007).

[19] D. D. Solnyshkov, I. A. Shelykh, M. M. Glazov, G. Malpuech, T. Amand, P. Renucci, X. Marie, and A. V. Kavokin, Semiconductors 41, 1080 (2007).

[20] F. Tassone and Y. Yamamoto, Phys. Rev. B 59, 10830 (1999).

[21] A. Thilagam, Physica (Amsterdam) 262B, 390 (1999).

[22] M. Vladimirova, S. Cronenberger, D. Scalbert, K. V. Kavokin, A. Miard, A. Lemaître, J. Bloch, D. Solnyshkov, G. Malpuech, and A. V. Kavokin, Phys. Rev. B 82, 075301 (2010).

[23] A. S. Brichkin, S. I. Novikov, A. V. Larionov, V. D. Kulakovskii, M. M. Glazov, C. Schneider, S. Höfling, M. Kamp, and A. Forchel, Phys. Rev. B 84, 195301 (2011). 